\title{
Effects of the Gas Outlet Duct Length on the Performance of Cyclone Separators
}

\author{
Bingbing Qiu ${ }^{1,2 *}$, and Wenke $\mathrm{Li}^{2}$ \\ ${ }^{1}$ School of Environmental Science and Engineering, Tianjin University, Tianjin 300072, China; \\ ${ }^{2}$ School of Energy and Environment, Anhui University of Technology, Ma' Anshan 243000, Anhui, China
}

\begin{abstract}
The numerical simulation of the cyclone separator was carried out by CFD software. The effects of the gas outlet duct length on the pressure drop and separation efficiency were discussed. The gas phase is used as a continuous medium, and the RNG k- $\epsilon$ turbulence model is used to simulate the flow field. Using the particle phase as a discrete system, a random orbital model is used to calculate the orbit of the particle based on the calculated flow field. The simulation results show that the flow field in the cyclone separator is anisotropic. When the inlet velocity is constant, the pressure drop of cyclone separators increases with the increase of gas outlet duct length. The longer gas outlet duct can limit the inflowing gas, so that there is enough time to establish uniform rotating flow. It helps stabilize the spiral airflow and improve the separation performance of cyclone separator.
\end{abstract}

\section{Introduction}

At present, the environmental pollution is serious. Dust particle is one of the major hazards that plague the safety production and seriously threaten human health. Flue gas is one of the main causes of dust generation. Currently, dust removal equipment is mainly used to reduce emissions. Cyclone separator is a dust removal equipment which separates particles from airflow mainly by centrifugal force. Cyclone separator is widely used in gas-solid separation equipment in the fields of chemical, metallurgy, refining and air pollution control due to its unique separation characteristic, low operating cost and easy maintenance. In industrial production, the performance of cyclone separator is affected by many factors, such as volume flow, particle size, and structural parameters.

Many scholars have carried out a lot of research on the separation performance of cyclone separators. Wang et al. [1] conducted experiments on cyclone separators with different gas outlet duct length. The results show that gas outlet duct length of the cyclone separator is an important factor affecting the separation efficiency and pressure drop. Cortés et al. [2] have studied the effect of axial inlet on cyclone separator. The results show that cyclone separator with axial inlet has better separation efficiency. Elsayed et al. [3] made a lot of theoretical research and numerical calculation on the separation performance of cyclone separators through empirical modeling and numerical simulation. Hoffmann et al. [4] analyzed the influence of the central pipe on the flow field of the cyclone separator. By changing the height and diameter of the central pipe, the optimal central pipe size was obtained.
Although the structural parameters of the cyclone separator, such as the influence of the length of gas outlet duct on the separation performance, have been experimentally studied, there are few studies on theoretical and numerical calculations. Compared with the industrial experiment, it is limited by the objective factors such as long production cycle, high production cost, and fixed structure parameters. The numerical simulation shows the advantages of the research and prediction. The CFD software is used to simulate the cyclone separator in this study. The influence of the gas outlet duct length on the pressure drop and separation efficiency were discussed. It is of great significance to the design and optimization of the cyclone separator structure.

\section{Mathematical models}

\subsection{Numerical simulation methods}

In the calculation, the gas phase is a continuous medium, and the RNG k- $\varepsilon$ turbulence model is selected. A random orbital model is used to describe the particle phase. The calculation method uses the SIMPLEC algorithm [5]. The continuity and the Reynolds average Navier-Stokes equations for a general incompressible flow are given as [6],

$$
\frac{\partial \rho u_{i}}{\partial x_{i}}=0
$$

\footnotetext{
* Corresponding author: qiliwei@ahut.edu.cn
} 


$$
\frac{\partial \rho u_{i}}{\partial t}+\frac{\partial}{\partial x_{j}}\left(\rho u_{i} u_{j}\right)=-\frac{\partial p}{\partial x_{i}}+\frac{\partial}{\partial x_{j}}\left[u\left(\frac{\partial u_{i}}{\partial x_{j}}+\frac{\partial u_{j}}{\partial x_{i}}\right)\right]
$$

In Eq. (2), $\mathrm{u}_{\mathrm{i}}$ is the velocity component and $\mathrm{P}$ is the static pressure, $\mathrm{xi}$ is the coordinate system, $\mathrm{v}$ is the kinematic viscosity and pis the gas density.

\subsection{Cyclone geometry and grids}

The cyclone inlet of the common cyclone separator is different from the tangential direction of the outer cylinder. The circular section between the inner and outer cylinders of the cyclone is used as the inlet, and the velocity direction is almost perpendicular to the section. The coordinate origin is located at the bottom center of the cylindrical section. The column section of the cyclone separator is long $950 \mathrm{~mm}$, the total length of the cone part is $270 \mathrm{~m}$. The rotor blade is evenly distributed in the cyclone separator, the right spin, the spiral angle is 35 degrees, and the rotation length of the swirling blade is $280 \mathrm{~mm}$. Figure 1 shows the physical model and local mesh model of the cyclone separator.

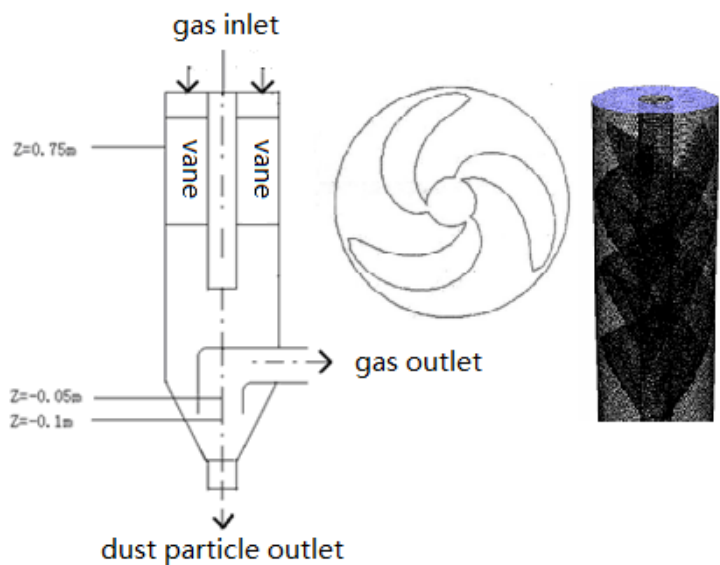

Fig. 1 The physical model and local mesh model of the cyclone separator.

\subsection{Simulations setup}

The boundary conditions of the gas phase set the inlet to be the velocity inlet and the outlet to use the free outlet. At the overflow outlet the static pressure was prescribed and all the cyclone walls were considered as no-slip boundaries.

At the solid surfaces, particles were reflected considering a perfectly elastic collision. Particles that crossed the overflow face were considered escaped. As in [5] particles that touched the cone bottom were considered collected.

\section{Results and discussion}

Gas outlet duct length is one of the important parameters of the cyclone separator. This study focuses only on the influence of overflow duct length on cyclone performance. The dimensions of the cyclone are displayed in Fig. 2. Numerical simulations were used to simulate the gas outlet duct length at $30 \mathrm{~mm}, 70 \mathrm{~mm}$ and
$110 \mathrm{~mm}$, respectively, and their effects on the performance of the cyclone were analyzed.

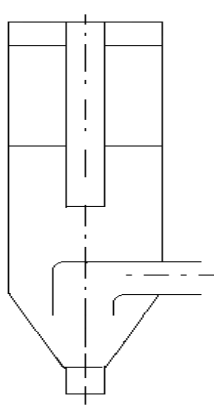

(a)

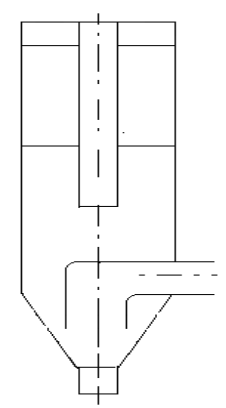

(b)

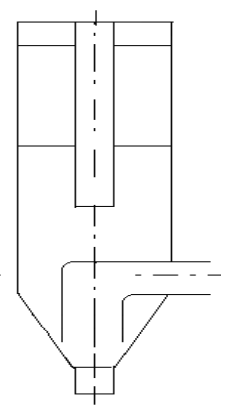

(c)
Fig. 2 Schematic diagram of different gas outlet duct lengths a) $30 \mathrm{~mm}$; b) $70 \mathrm{~mm}$; c) $110 \mathrm{~mm}$.

\subsection{Effect of different gas outlet duct lengths on cyclone pressure drop}

Different gas outlet duct lengths have different effects on the pressure drop of cyclone separators. The structural models of these three cases are established respectively for numerical simulation. When the inlet velocity is $3.24 \mathrm{~m} / \mathrm{s}$ (rated volume flow is $300 \mathrm{~m}^{3} / \mathrm{h}$ ), the total pressure and pressure drop of the inlet and outlet of the cyclone separator are obtained. The relationship between the pressure drop and gas outlet duct length is shown in Fig. 3.

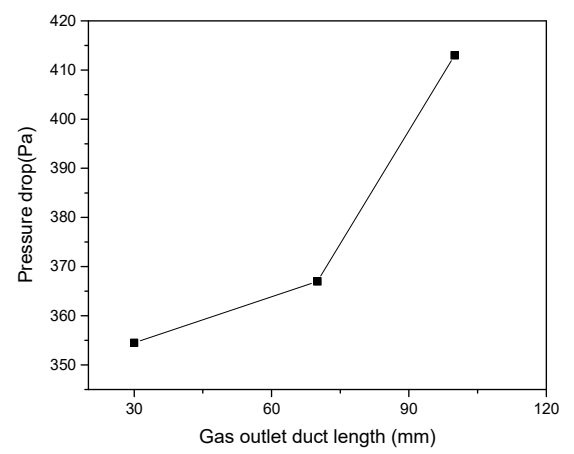

Fig.3 Effect of different gas outlet duct lengths on cyclone pressure drop

Fig. 3 shows that under the condition that the inlet speed of the airflow is constant, when the gas outlet duct length increases, the total inlet pressure and the total outlet pressure of the airflow increase, and the pressure loss also increases. This is consistent with the results of the literature [5]. This is mainly due to the increase of gas outlet duct length, which makes the flow of the gas longer, and the contact area between the solid wall of the cyclone and the air flow increases, and the pressure loss increases owing to the friction.

\subsection{Effect of different gas outlet duct lengths on cyclone separation efficiency}

Different gas outlet duct lengths have a great influence on the separation efficiency of the particles in the cyclone. The particle separation efficiency of gas outlet 
duct at different lengths is obtained by numerical simulation, and the relationship is shown in Fig. 4.

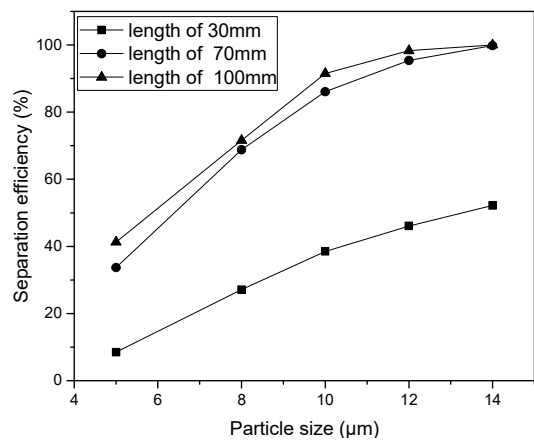

Fig. 4 Effect of gas outlet duct length on separation efficiency with different particle size

Fig. 4 shows that the separation efficiency of solid particles in cyclone separators increases with the increase of gas outlet duct length. This is mainly because when the length of the gas outlet duct is increased, it can limit the inflowing gas, so that there is enough time to establish uniform rotational flow. In addition, when the gas outlet duct is long, the dusty gas that originally enters the gas outlet duct directly from the inlet will also rotate in accordance with the ideal route. This is consistent with the results of the [2]. This indicates that a longer gas outlet duct can stabilize the spiral flow and improve the separation performance of the cyclone.

\subsection{Velocity distribution of the flow field}

The velocity field and particle trajectory of the cyclone with an optimized gas outlet duct length of $70 \mathrm{~mm}$ were simulated. The instantaneous velocity vector of the three typical cross sections is shown in Fig. 5.

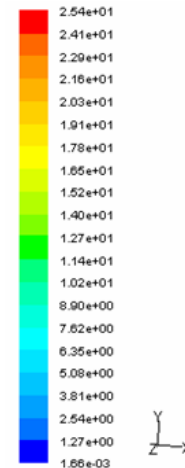

$2.54 \mathrm{e}+01$
$2.41 \mathrm{e}+01$
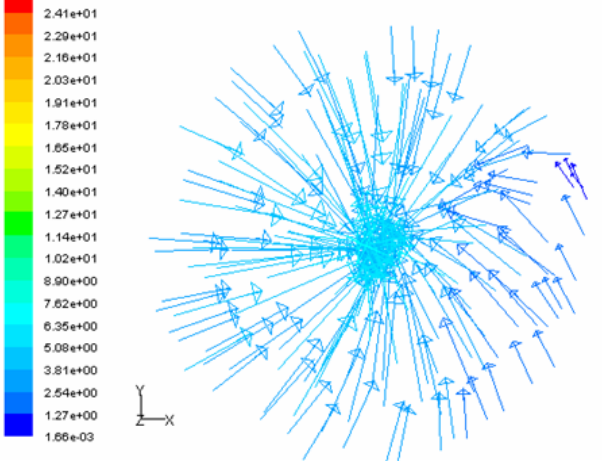

(b)
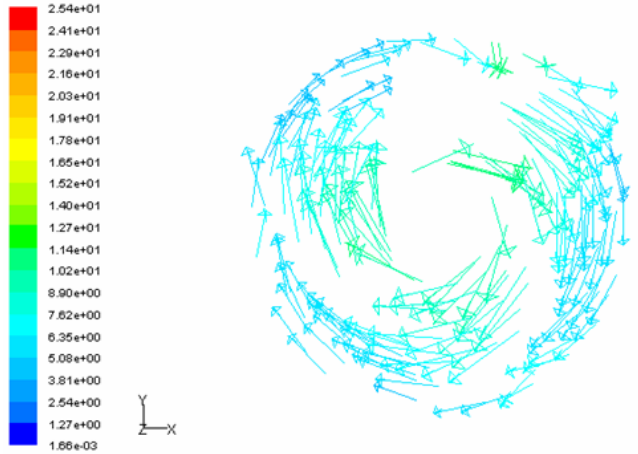

(c)

Fig. 5 Velocity vector diagram of cross section of (a) $Z=-$ $0.05 \mathrm{~m}$, (b) $Z=-0.10 \mathrm{~m}$ and (c) $Z=0.75 \mathrm{~m}$.

The velocity vector of the flow field shows the flow regime inside the cyclone. The results show that the axial entry of the gas into the cyclone produces a swirling flow due to the action of the swirling vanes. In the outer (outer vortex) of the swirl, the gas moves downward. When the air reaches the bottom of the cyclone, there is basically no flow of air at the exhaust port, which moves up in the middle of the cyclone and move upward (inner vortex) in the same direction of rotation [6].

\subsection{Particle trajectory tracking}

The trajectories of particles of different particle sizes in the cyclone are different, and it has a certain randomness. In this study, five particle sizes were examined: $5 \mu \mathrm{m}$, $8 \mu \mathrm{m}, 10 \mu \mathrm{m}, 12 \mu \mathrm{m}$ and $20 \mu \mathrm{m}$. Fig. 6 shows the trajectory of particles as a function of time.

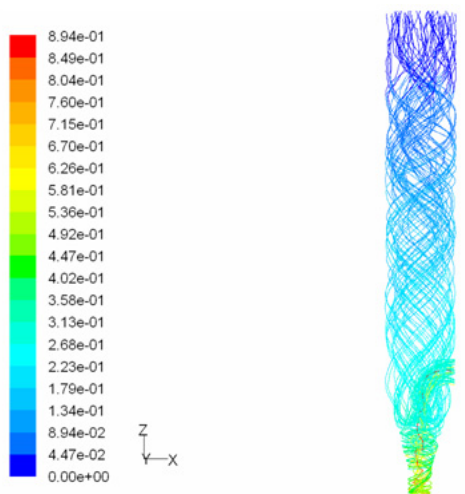

(a)
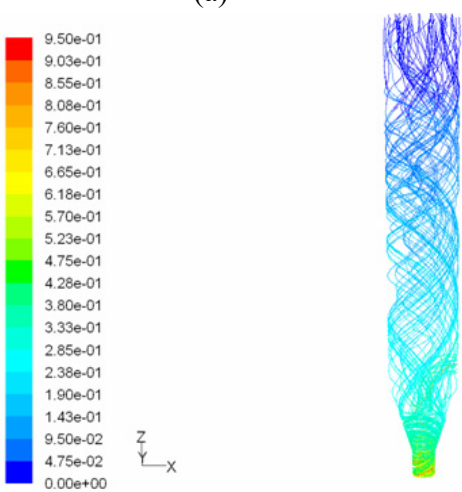

(b) 

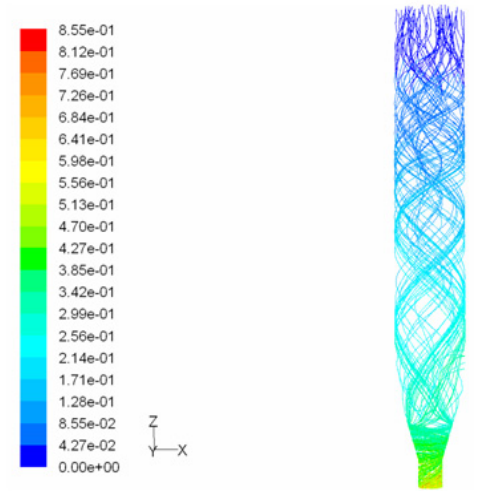

(c)
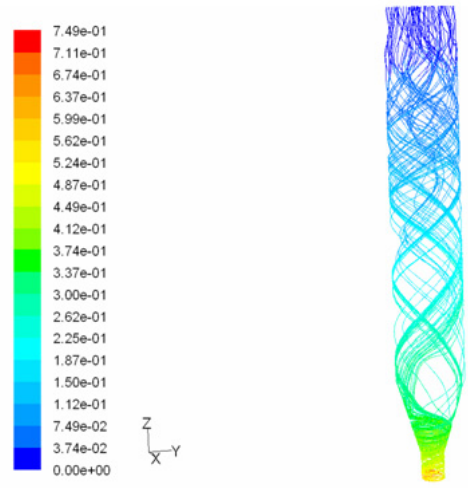

(d)
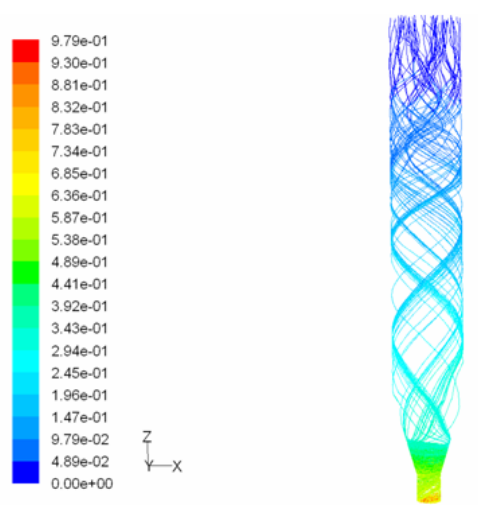

(e)

Fig. 6 Trajectories of particles of (a) $5 \mu \mathrm{m}$, (b) $8 \mu \mathrm{m}$, (c) $10 \mu \mathrm{m}, \quad$ (d) $12 \mu \mathrm{m}$ and (e) $20 \mu \mathrm{m}$

The particles of the five particle sizes are uniformly injected from the inlet section. It can be seen from their trajectories that particles with a smaller particle size have a smaller rotation pitch and a larger number of rotations in the cyclone, and it takes a long time to leave the cyclone (escape or trap). The particle with larger particle size has a larger rotating pitch, and the number of rotating turns in the cyclone is higher. After entering the cyclone, it is quickly turned to the wall, and then the wall is turned downward to enter the ash hopper. This is consistent with the results of prior literature $[7,8]$.

\section{Conclusions}

Too short gas outlet duct can cause the core of the swirl to bend, making it unstable. This also easily causes short circuit of the airflow, resulting in reduced dust removal efficiency. But its manufacturing cost is less, inspection and maintenance are more convenient, and total pressure loss is reduced. When the gas outlet duct is too long, the cost of manufacturing is higher and the inspection and maintenance are difficult. It may increase the pressure loss because of friction with the tube wall, and the vortex flow can be deformed because of the decrease of the rotating speed on the wall. Therefore, these factors should be fully considered and a reasonable gas outlet duct length should be selected. The particles move downward with the swirling airflow, and the number of rotations gradually decreases as the particle size increases. A certain length of gas outlet duct can reduce the residence time of particles, thus promoting the trapping effect of cyclone separators. At the same time, the separation efficiency increased with the increase of particle size.

\section{Acknowledgment}

This research thanks for the financial supported by Natural Science Research of Anhui.

\section{References}

1. B. Wang, D.L. Xu, K.W. Chu, A.B.Yu, Appl. Math. Model. 30, 1326(2006)

2. C. Cortés, A.Gil, Prog. Energ. Combust. 33, 409(2007)

3. K. Elsayed, C. Lacor, Powder Technol. 217, 84(2012)

4. A.C. Hoffmann, M.D. Groot, W. Peng, H.W.A. Dries, J. Kater, AICHE J. 47, 2452(2010)

5. K. Elsayed, Powder Technol., 269, 409(2015)

6. H. Safikhani, A. Hajiloo, M.A. Ranjbar, Comput. Chem. Eng. 35, 1064(2011)

7. Y. Su, A. Zheng, B. Zhao, Powder Technol. 210, 293(2011)

8. F.J.D. Souza, R.D.V. Salvo, D.A.D.M. Martins, Sep. Purif. Technol. 94, 61(2012) 